Azurin for Biomolecular Electronics: a Reliability Study

This content has been downloaded from IOPscience. Please scroll down to see the full text. 2005 Jpn. J. Appl. Phys. 446864

(http://iopscience.iop.org/1347-4065/44/9R/6864)

View the table of contents for this issue, or go to the journal homepage for more

Download details:

IP Address: 132.239.1.230

This content was downloaded on 06/09/2015 at 02:13

Please note that terms and conditions apply. 
(C) 2005 The Japan Society of Applied Physics

\title{
Azurin for Biomolecular Electronics: a Reliability Study
}

\author{
Alessandro Bramanti*, Pier Paolo Pompa, Giuseppe Maruccio, Franco Calabi, Valentina Arima, \\ Roberto Cingolani, Stefano CoRni ${ }^{1}$, Rosa Di Felice ${ }^{1}$, Francesca De RienzO ${ }^{2}$ and Ross RinAldi \\ National Nanotechnology Laboratory (NNL) of INFM, Distretto Tecnologico, University of Lecce, Via per Arnesano, km 5, I-73100 Lecce, Italy \\ ${ }^{1}$ INFM Center on nanoStructures and bioSystems at Surfaces (S3), Modena, Italy \\ ${ }^{2}$ Dipartimento di Chimica and INFM-S3, Università di Modena e Reggio Emilia, Via Campi 213/A, 41100 Modena, Italy
}

(Received March 1, 2005; accepted April 27, 2005; published September 8, 2005)

The metalloprotein azurin, used in biomolecular electronics, is investigated with respect to its resilience to high electric fields and ambient conditions, which are crucial reliability issues. Concerning the effect of electric fields, two models of different complexity agree indicating an unexpectedly high robustness. Experiments in device-like conditions confirm that no structural modifications occur, according to fluorescence spectra, even after a 40-min exposure to tens of $\mathrm{MV} / \mathrm{m}$. Ageing is then investigated experimentally, at ambient conditions and without field, over several days. Only a small conformational rearrangement is observed in the first tens of hours, followed by an equilibrium state. [DOI: 10.1143/JJAP.44.6864]

KEYWORDS: biomolecular electronics, protein devices, high electric fields, folding, device reliability, theoretical models, solidstate measurements

Azurin is a metalloprotein involved in electron transfer in $P$. aeruginosa. ${ }^{1,2)}$ Its redox active center consists of a $\mathrm{Cu}$ atom coordinated by five ligands, arranged in a trigonal bipyramidal geometry. The $\mathrm{Cu}(\mathrm{I})$ and $\mathrm{Cu}(\mathrm{II})$ states show minimal structural differences, and a consequently low reorganization energy, which favors redox-mediated electron transfer. Thanks to such characteristics, azurin has recently been employed with success as the active element of rectifying (diode-like) and modulating (transistor-like) biomolecular devices. ${ }^{3-6)}$ Yet, two major objections are frequently raised against electronic applications of biomolecules in the solid state. First, the high electric fields applied to nanometric devices are indicated as potential sources of rapid molecular degradation. Second, biological macromolecules may not be structurally stable in ambient conditions (in air). In this work, the problem of reliability of azurinbased devices is addressed in both respects.

In nanometric devices, biomolecules may be subjected to electric fields of the order of $10^{7} \mathrm{~V} / \mathrm{m}$. Molecular dynamics simulations ${ }^{7)}$ already indicated that proteins may be able to resist the electric stress of devices without permanent structural alterations. Estimating the magnitude of the intra-molecular electric field of the protein gives an indication of its resilience to an applied field. To evaluate the average intensity of the inner electric field in azurin, a simplified analytical model was used as a first approximation. Previous calculations ${ }^{3,4)}$ had highlighted the presence of a strong electric dipole $(\approx 150 \mathrm{D})$ in azurin. By approximating the globular shape of azurin to a sphere of uniform polarization $p$, a well-known result of electrostatics states that the magnitude of the inner (uniform) electric field is

$$
|E|=\frac{p}{\varepsilon_{0}\left(2 \varepsilon_{\mathrm{r}}+1\right)},
$$

where $\varepsilon_{0}$ is the permittivity of vacuum and $\varepsilon_{\mathrm{r}}$ is the relative permittivity of the surrounding dielectric. The integration of $p$ over the volume must yield the total dipole moment $P$, therefore:

$$
p=\frac{P}{(4 / 3) \pi R^{3}} \Rightarrow|E|=\frac{3 P}{4 \pi \varepsilon_{0} R^{3}\left(2 \varepsilon_{\mathrm{r}}+1\right)} .
$$

*E-mail: alessandro.bramanti@st.com. A. Bramanti is presently with STMicroelectronics, Research Unit of Lecce.
By assuming the sphere radius $R=2 \mathrm{~nm}$, one obtains $|E|=1.1 \times 10^{7} \mathrm{~V} / \mathrm{m}$. Such magnitude is comparable to the intensity of the applied fields; however, it is an average estimate, which assumes the intra-molecular field to be uniform in its intensity and orientation. We remark that in eqs. (1) and (2) we employed the vacuum dielectric constant. Taking into explicit account the protein polarization properties would require the use of a relative dielectric constant between 2 and 4, as found for dry protein powders. ${ }^{7-9)}$ This, however, would change the evaluated field by less than an order of magnitude.

To assess the validity of this simple model, we carried out a more refined calculation, evaluating the inner fields from the molecular electrostatic potential (MEP). The MEP of azurin from $P$. aeruginosa in solution was computed by solving the linearized Poisson-Boltzmann equation for a given charge distribution, ${ }^{10}$ corresponding to the $\mathrm{X}$-ray structure (PDB file 4azu at http://pdb.gmd.de). The University of Houston Brownian Dynamics (UHBD) package ${ }^{11)}$ was employed for the calculations. ${ }^{12)}$ The field was finally derived as the spatial gradient of the potential, on the same cubic computational grid, with a spacing of $1 \AA$. Such a simple model may not be accurate as to point values, but can give a reliable estimate. Figure 1 reports the cumulative frequency distribution of the field magnitude, i.e., the probability of finding a field magnitude smaller than a given value, inside the intra-molecular volume. ${ }^{13)}$ The field is estimated to be larger than $10^{7} \mathrm{~V} / \mathrm{m}$ over nearly the whole volume, and larger than $10^{8} \mathrm{~V} / \mathrm{m}$ in about $20 \%$ of the space (average magnitude $|E|=4 \times 10^{9} \mathrm{~V} / \mathrm{m}$ ). Our results are in agreement with those obtained by Callis and Burgess, though these authors used a different method based on the consistence valence force field and restricted their estimate to the region around Trp48. ${ }^{14)}$ This residue is responsible for azurin fluorescence, which is exactly the property probed in this work to test the resilience of the protein layer to typical device external fields (see below). Moreover, a fitting of vibrational Stark spectroscopic data (for proteins different from azurin) also gave comparable values. ${ }^{15)}$ Such high intrinsic electric field within the protein volume (up to two orders of magnitude higher than the field applied in devices) suggests that the device environment acts only as a minor perturbation to the native conditions, and does not induce 


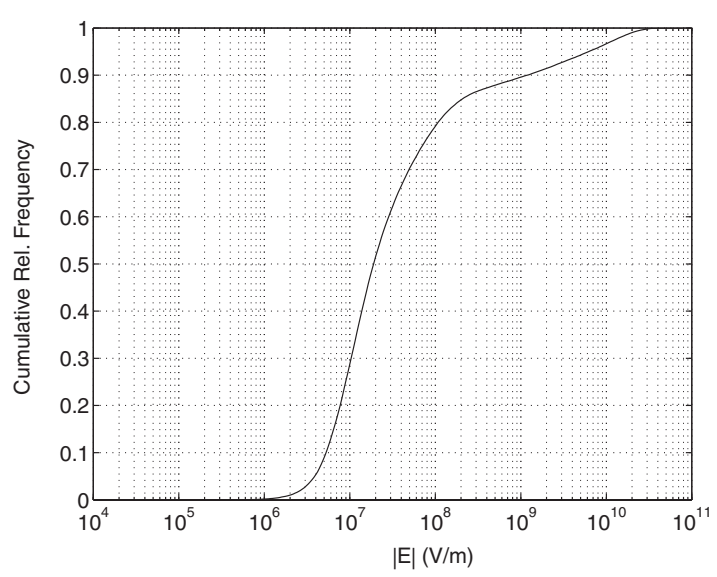

Fig. 1. Cumulative frequency distribution of the electric field magnitude inside the molecule (semilogarithmic scale). The relevant range is from $10^{6}$ to $10^{10} \mathrm{~V} / \mathrm{m}$.

loss of functionality.

The much smaller value found in the analytical model is explained by observing that, in the uniformly polarized sphere, the field sums up throughout the volume, to give the total dipole moment. Instead, the true field components have variable orientation, and can thus assume, from point to point, far higher absolute values. Indeed, their signed average values, as derived from the MEP calculation, are: $E_{x}=5 \times 10^{5} \mathrm{~V} / \mathrm{m}, E_{y}=-1 \times 10^{7} \mathrm{~V} / \mathrm{m}$ and $E_{z}=-3 \times$ $10^{5} \mathrm{~V} / \mathrm{m}$, in agreement with those obtained analytically. Because of the non-uniform character of real fields, the analytical model provides a lower boundary for the resilience of azurin. Since a similar argument is likely to be applicable to a wide class of proteins, simple analytical models may be useful for a gross evaluation of molecular resilience.

In order to verify the predicted robustness of azurin, its folding properties under external electric fields were investigated by intrinsic fluorescence spectroscopy. ${ }^{16)}$ Protein molecules were immobilized onto a $\mathrm{SiO}_{2}$ substrate, ${ }^{17)}$ on which gold interdigitated electrodes were present. ${ }^{18)}$ The inter-electrode separation, in the order of microns, implies the attainment of fields up to $10^{7} \mathrm{~V} / \mathrm{m}$ by applying tens of volts. Finite element method (FEM) simulations demonstrated that the electric field between pairs of electrodes is practically uniform (data not shown). Furthermore, the electrodes were spread over a $1 \times 1 \mathrm{~mm}^{2}$ area, thus allowing us to detect the (otherwise weak) fluorescence signal. ${ }^{19)}$ To the authors' knowledge, these are the first experiments of this kind, as to the high intensity of the fields applied in a solid state environment, at room temperature and atmospheric pressure. The presence of azurin after deposition was checked by tapping mode atomic force microscopy (TMAFM, Fig. 2). The profiles indicate a different relative height between top and bottom, after the azurin deposition. This means that the bottom of the sample has been covered by a thicker molecular layer than the top of the electrodes and so that most azurin has fallen in between the electrodes themselves (it should be noted that the heights indicated on the $y$ axes of the figure are not absolute, i.e., they have not been taken with respect to the same reference in the two profiles). Moreover, the fluorescence intensity did not vary significantly over different regions of the substrate, indicating that the molecular layer was substantially homogeneous.
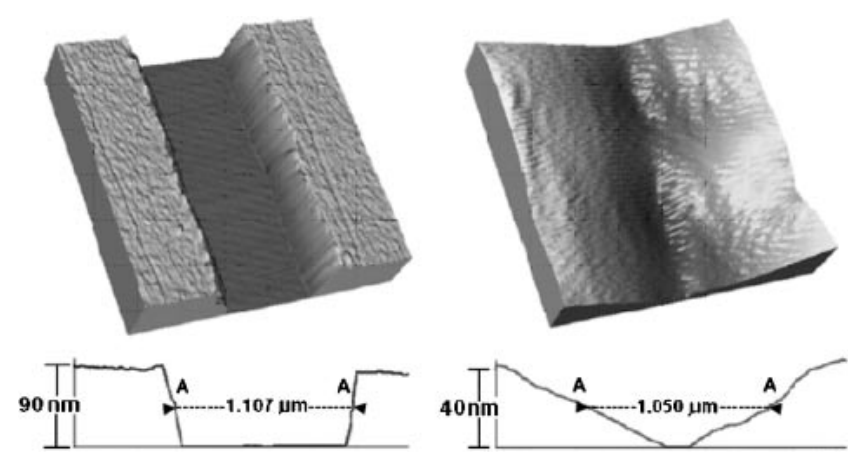

Fig. 2. AFM topography of the electrodes, before (left) and after (right) the deposition of azurin, in 3D and cross-sectional representations. The deposition of a layer of softer matter is clear from the right side topography. The decreased relative top-to-bottom height in the corresponding profile indicates that most molecules lie in between the electrodes.

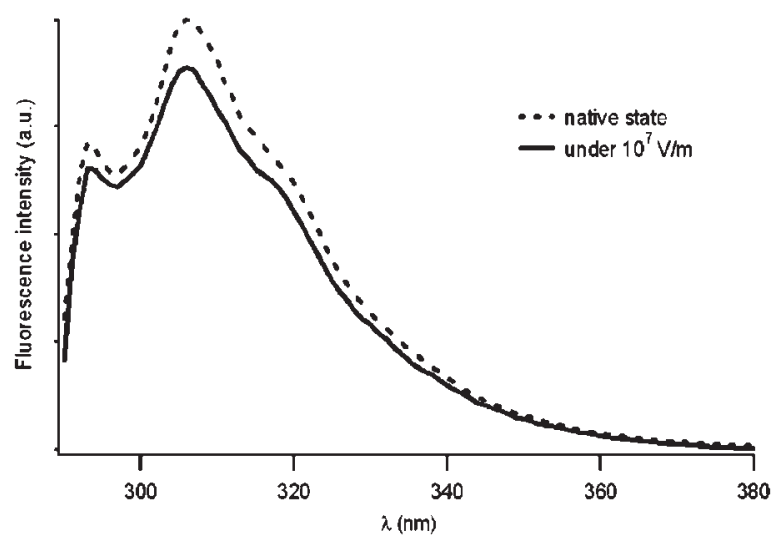

Fig. 3. Fluorescence spectra for $\mathrm{Zn}$-azurin in the native state and under an electric field typical of the experimental device conditions. It is evident that the protein preserves its characteristic fluorescence spectrum even after $40-\mathrm{min}$ exposure to $10^{7} \mathrm{MV} / \mathrm{m}$ electric field.

The fields were applied for periods up to $40 \mathrm{~min}$. Figure 3 compares the photoluminescence (PL) spectra of azurin in the native state and under field. Clearly, there is no change at all, consistently with our theoretical predictions.

The second reliability issue concerns ageing of azurin in air, at ambient conditions. Some of us already reported experimental evidence ${ }^{20,21)}$ that immobilization of azurin in a solid state monolayer, in air, does not necessarily lead to protein denaturation. As an additional proof, the ageing of azurin was monitored over periods longer than 600 hours, with zero external bias. Figure 4 reports the emission maximum $\lambda_{\max }$, full width at half maximum (FWHM), and integrated emission, all as function of time, starting from the solvent removal instant. It is evident that solid state proteins undergo modest conformational rearrangement. As seen in Fig. 4(a), only a slight red-shift of the azurin PL occurs, which brings $\lambda_{\max }$ to a maximum of $311-312 \mathrm{~nm}$, well below the typical broadband "red" emission of denatured azurin $\left(\lambda_{\max } \approx 355 \mathrm{~nm}\right)$. Most of this modification takes place within the first $7 \mathrm{~h}$, after which a further little rearrangement occurs on a time scale of $100 \mathrm{~h}$. This behavior is common to all the three spectral parameters plotted in Fig. 4. No other significant conformational changes are observed after the first $100 \mathrm{~h}$ for up to weeks under non-physiological conditions. It 

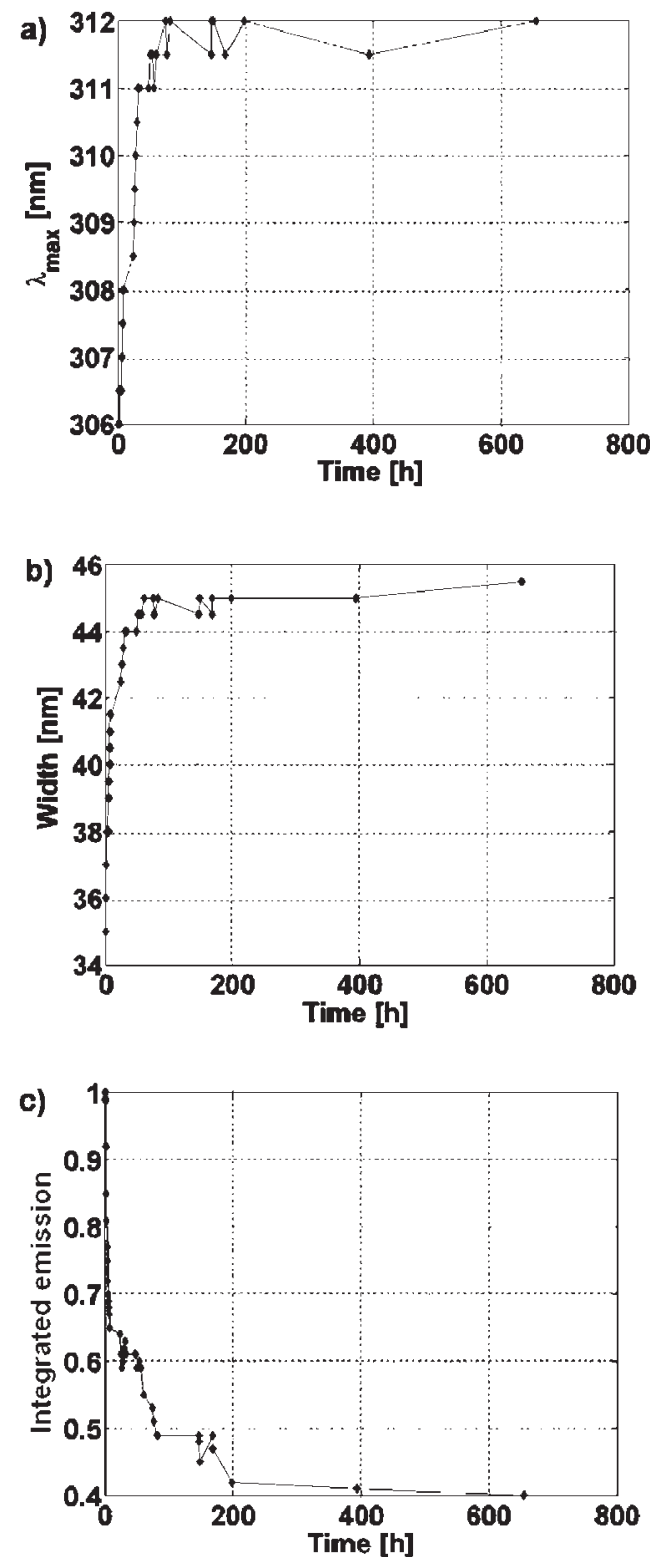

Fig. 4. Ageing of azurin at ambient conditions, over more than $600 \mathrm{~h}$ : (a) emission maximum, (b) full width at half maximum and (c) normalized integrated emission, as a function of time from the solvent removal. After a minor rearrangement over a few hours, azurin reaches a stable configuration.

seems that proteins at ambient conditions (room temperature, $50-60 \%$ relative humidity) reach a stable state, which may be attributed to the capability of azurin of retaining its hydration shell. It is worth noting that upon rehydration at the end of the investigated period, the PL spectrum typical of native azurin in solution is recovered, indicating the minor scale of the rearrangement on dehydration. This is the first experimental evidence of such effects.

In conclusion, azurin is found to be much more reliable a material than might have been expected. These results corroborate the use of this protein in biomolecular devices and, to some extent, reinforce the trust in this novel branch of electronics.

The authors thank Professor G. W. Canters (Leiden Institute of Chemistry, Leiden, NE) for azurin supplies, E. Molinari and P. Facci (INFM-S3) for discussions and critical reading of the manuscript, and E. D'Amone for technical support. This work was partly funded by the EC through project "SAMBA" and by MIUR-Italy through FIRBNOMADE.

1) E. T. Adman: Adv. Protein Chem. 42 (1991) 145.

2) A. G. Sykes: Adv. Inorg. Chem. 36 (1991) 377.

3) R. Rinaldi, A. Biasco, G. Maruccio, R. Cingolani, D. Alliata, L. Andolfi, P. Facci, F. De Rienzo, R. Di Felice and E. Molinari: Adv. Mater. 14 (2002) 1453.

4) R. Rinaldi, A. Biasco, G. Maruccio, V. Arima, P. Visconti, R. Cingolani, P. Facci, F. De Rienzo, R. Di Felice, E. Molinari, M. Ph. Verbeet and G. W. Canters: Appl. Phys. Lett. 82 (2003) 472.

5) R. Rinaldi, G. Maruccio, A. Biasco, P. Visconti, S. D'Amico and R. Cingolani: Ann. N.Y. Acad. Sci. 1006 (2003) 187.

6) R. Rinaldi and R. Cingolani: Physica E 21 (2004) 45.

7) D. Xu, J. C. Phillips and K. Schulten: J. Phys. Chem. 100 (1996) 12108.

8) S. Takashima and H. P. Schwan: J. Phys. Chem. 69 (1965) 4176.

9) S. C. Harvey and P. Hoekstra: J. Phys. Chem. 76 (1972) 2987.

10) The atomic charges and radii were assigned to each protein residue according to the empiric OPLS non-bonded parameter sets [W. L. Jorgensen and J. Tirado-Rives: J. Am. Chem. Soc. 110 (1988) 1657]. The $\mathrm{Cu}$-site residues were parameterized using a simplified charge distribution [F. De Rienzo, R. R. Gabdoulline, M. C. Menziani and R. C. Wade: Protein Sci. 9 (2000) 1439].

11) J. D. Madura, J. M. Briggs, R. C. Wade, M. Davis, B. A. Luthy, A. Ilin, J. Antonsiewicz, M. K. Bagheri, B. Scott and J. A. McCammon: Comput. Phys. Commun. 91 (1995) 57.

12) In the UHBD scheme, the solvent is implicitly taken into account in terms of the dielectric constant $\varepsilon$ (relative permittivity $\varepsilon_{\mathrm{r}}=78$, for water) and the ionic strength. The protonation states of the titratable amino acids were chosen consistently with the $\mathrm{pH}$ value of the solved 4azu azurin structure.

13) The core region of each atom, where divergencies make the field illdefined, were excluded from the statistics of the electric field values, with a simple procedure based on the identification in the UHBD computational grid of the eight nearest-neighbor grid-points of every atom.

14) P. R. Callis and B. K. Burgess: J. Phys. Chem. B 101 (1997) 9429.

15) E. S. Park, S. S. Andrews, R. B. Hu and S. G. Boxer: J. Phys. Chem. B 103 (1999) 9813.

16) To this aim, the zinc derivative of azurin was used, since the PL emission of the wild type copper protein is strongly quenched by the copper ion. This causes no loss of validity, though, since $\mathrm{Zn}$-azurin has a lower structural stability than $\mathrm{Cu}$-azurin.

17) A $20 \mu \mathrm{l}$ drop of $1.0 \mathrm{mg} / \mathrm{ml}$ protein solution (in $50 \mathrm{mM} \mathrm{NH} 4 \mathrm{Ac}$ buffer, $\mathrm{pH}$ 4.6) was cast deposited and incubated for $15 \mathrm{~min}$ at room temperature. Subsequently, the buffer was removed and samples dried under high purity nitrogen flow.

18) 500 interdigitated electrodes, covering an area of $1 \times 1 \mathrm{~mm}^{2}$ with a $2 \mu \mathrm{m}$ periodicity (electrode width: $1 \mu \mathrm{m}$, line spacing: $1 \mu \mathrm{m}$ ) were fabricated on thermally oxidized silicon wafers, using standard photolithographic techniques by e-beam metal evaporation $(\mathrm{Cr} / \mathrm{Au})$ followed by lift-off in acetone bath.

19) Fluorescence spectra were recorded in photon counting mode, (excitation source: $450 \mathrm{~W}$ Xenon lamp; double monochromators used both in excitation and emission). For measurements in solution, the emission was observed at right angles to the excitation. Photoluminescence spectra of protein-free control samples were subtracted from the experimental samples to correct for background. For solidstate luminescence monitoring, the exciting beam was focused on the film with $30^{\circ}$ incidence angle, and the front surface emission was collected at $90^{\circ}$ from the excitation. The excitation intensity was very low (in the order of $100 \mathrm{~W} / \mathrm{cm}^{2}$ ) with shorter-than-1-min acquisition times. Thus, fluorescence intensity losses both over single and repeated measurements were negligible, excluding appreciable Trp photodegradation during acquisition.

20) P. P. Pompa, A. Biasco, R. Cingolani, R. Rinaldi, M. Ph. Verbeet and G. W. Canters: Phys. Rev. E 69 (2004) 032901.

21) P. P. Pompa, A. Biasco, V. Frascerra, F. Calabi, R. Cingolani, R. Rinaldi, M. Ph. Verbeet, E. De Waal and G. W. Canters: J. Chem. Phys. 121 (2004) 10325. 